\title{
STABLE PROCESSES HAVE THORNS
}

\author{
BY KRZYSZTOF BURDZY ${ }^{1}$ AND TADEUSZ KULCZYCKI ${ }^{2}$ \\ University of Washington, Polish Academy of Sciences and \\ Wroctaw University of Technology
}

Let $X(t)$ be the symmetric $\alpha$-stable process in $\mathbf{R}^{d}, \alpha \in(0,2)$, $d \geq 2$. For $f:(0,1) \rightarrow(0, \infty)$ let $D(f)$ be the thorn $\left\{x \in \mathbf{R}^{d}: x_{1} \in(0,1)\right.$, $\left.\left|\left(x_{2}, \ldots, x_{d}\right)\right|<f\left(x_{1}\right)\right\}$. We give an integral criterion in terms of $f$ for the existence of a random time $s$ such that $X(t)$ remains in $X(s)+\bar{D}(f)$ for all $t \in[s, s+1)$.

1. Introduction. Let $X(t)$ be the symmetric $\alpha$-stable process in $\mathbf{R}^{d}$, that is, the Markov process with independent and homogeneous increments and the characteristic function of the form $E^{x} e^{i \xi X(t)}=e^{i x \xi-t|\xi|^{\alpha}}, x, \xi \in \mathbf{R}^{d}, t \geq 0$. We will consider only pure jump multidimensional stable processes, that is, we will assume that $d \geq 2$ and $\alpha \in(0,2)$. We will denote by $\Omega$ the probability space on which $X(t)$ is defined. We may and will assume that all paths $t \rightarrow X(t, \omega), \omega \in \Omega$, are right continuous with left limits [3].

Let $f:(0,1) \rightarrow(0, \infty)$ be a nondecreasing left continuous function which satisfies $\lim _{r \rightarrow 0^{+}} f(r)=0$. For $x=\left(x_{1}, x_{2}, \ldots, x_{d}\right) \in \mathbf{R}^{d}$ we will write $\tilde{x}=$ $\left(x_{2}, \ldots, x_{d}\right)$. We define a thorn $D(f)$ as follows:

$$
D(f)=\left\{x \in \mathbf{R}^{d}: x_{1} \in(0,1),|\tilde{x}|<f\left(x_{1}\right)\right\} .
$$

The closure of $D(f)$ will be denoted by $\bar{D}(f)$.

The main result of our paper is the following. If a thorn $D(f)$ is not very thin then $X(t)$ has $D(f)$-thorn points at some random times $s$, that is, the trajectory stays for $t \in[s, s+1)$ within the (translated) closed thorn whose sharp point is at $X(s)$. A formal statement of the result is contained in Theorem 1.1.

We will fix some $t_{0}>0$ and often suppress it in the notation. Let

$$
\begin{aligned}
& A(f)=\{\omega \in \Omega: \text { there exists time } s=s(\omega) \geq 0 \text { such that } \\
& \left.\qquad X(t, \omega) \in X(s, \omega)+\bar{D}(f) \text { for all } t \in\left[s, s+t_{0}\right)\right\}, \\
& I(f)=\int_{0}^{1 / 2} \frac{(f(r))^{d+\alpha-1}}{r^{d+\alpha}} d r .
\end{aligned}
$$

Received February 2001; revised December 2001.

${ }^{1}$ Supported in part by NSF Grant DMS-00-71486.

${ }^{2}$ Supported in part by the Foundation for Polish Science and by KBN Grant 2 P03A 02816.

AMS 2000 subject classifications. 60G17, 60G52.

Key words and phrases. Symmetric stable process, local properties of trajectories, thorn points, thorns. 
THEOREM 1.1. For any $t_{0}>0$, the probability of $A(f)$ is equal to:

(i) 1 if $I(f)=\infty$,

(ii) 0 if $I(f)<\infty$.

It is easy to check that if $f(r)=r|\log r|^{\beta}$ then $I(f)=\infty$ if and only if $\beta \geq-1 /(d+\alpha-1)$. Local properties of stochastic processes are often described by comparing process trajectories with "regularly varying" functions. Our integral criterion allows us to consider very rough thorns, for example, if $f$ is nondecreasing and for some constant $c>0$, there exist arbitrarily small $r>0$ with $f(r)>c r$, then $I(f)=\infty$ and so the stable process has thorn points defined by such functions $f$.

If $f(r)=c r$ for some constant $c>0$, the set $D(f)$ is a cone. Brownian motion has cone points for some but not all values of $c>0$. Cone points were discussed for the first time in [10], [14] and [19]. See [2] for a review of recent results on Brownian path properties related to cone points.

The existence of cone points on Brownian paths is a nontrivial result which requires several technical ingredients developed and collected by several authors over the years. Here is a sketch of a proof of the existence of cone points for stable processes using a "soft" argument. Fix any $c>0$ and let $f(r)=c r$. Since $X(t)$ has jumps, with positive probability there will be a jump time $s=s(\omega)$ such that $\{X(t, \omega), 0 \leq t<s\} \subset X(s, \omega)+D(f)$. Standard arguments based on scaling and the strong Markov property can be used to strengthen this claim; one can show that for every $\varepsilon>0$, with probability 1 , there exists $s=s(\omega) \in(0, \varepsilon)$ with $\{X(t, \omega), 0 \leq t<s\} \subset X(s, \omega)+D(f)$. Fix an arbitrarily small $p>0$. Let $\delta_{1}>0$ be so small that with probability greater than $1-2^{-1} p$, there is a jump time $s=s(\omega) \in\left(0,1-2^{-1}\right)$, such that $|X(s, \omega)-X(s-, \omega)| \geq \delta_{1}$ and $\{X(t, \omega), 0 \leq t<s\} \subset X(s, \omega)+D(f)$. Let $S_{1}$ be the smallest $s$ with this property (or $\infty$, if there is no such $s$ ). Suppose that $S_{1}<\infty$. Since $S_{1}$ is a stopping time, by the strong Markov property one can find $\delta_{2}>0$ so small that with probability greater than $1-2^{-2} p$, there is a jump time $s=s(\omega) \in\left(S_{1}, 1-2^{-2}\right)$, such that $|X(s, \omega)-X(s-, \omega)| \geq \delta_{2}$ and $\left\{X(t, \omega), S_{1} \leq t<s\right\} \subset X(s, \omega)+D(f)$. We let $S_{2}$ be the smallest $s>S_{1}$ with this property $\left(S_{2}=\infty\right.$, if there is no such $\left.s\right)$. By induction, with probability not less than $1-p$, we will have a sequence of stopping times $S_{k}$ increasing to $S_{\infty} \leq 1$. Elementary geometry shows that $\left\{X(t, \omega), 0 \leq t<S_{\infty}\right\} \subset X\left(S_{\infty}, \omega\right)+D(f)$. Let $\{\tilde{X}(t), t \in[0,1]\}$ be the cadlag version of $\{X(1-t), t \in[0,1]\}$. The process $\tilde{X}(t)$ has the same distribution as $X(t)$. It is elementary to check that $\tilde{X}(t)$ has a cone point at $s=1-S_{\infty}$, that is, the event $A(f)$ holds for $\tilde{X}$ with $s=1-S_{\infty}$ and some $t_{0}>0$. Since $p>0$ is arbitrary, cone points exist with probability 1 .

The soft proof of existence of cone points for stable processes had indicated to us the existence of thorn points before we started our project. 
We would like to point out another case when a "soft" (although clever) argument can yield a result whose continuous counterpart is quite difficult. According to Proposition 3 of [17], there is no uniform modulus of continuity for a "generic" Lévy process, which would apply at all times $t$ at which the process does not have a jump.

Our study of thorn points has been motivated in several ways. First of all, the main result seems to have some interest of its own. Second, studying local path properties of stochastic processes is a test for the corresponding potential theoretic methods. Finally, it was shown in [18] that Brownian cone points are related to reflected Brownian motion in a wedge. Hence, one may expect that thorn points for stable processes might be related to "censored" stable processes, the subject of a forthcoming paper [7].

One may ask whether stable processes have thorn points with arbitrary (random) directions unlike those in Theorem 1.1. The corresponding problem for Brownian cone points is not very hard because cones with small angles fit into some cones with larger angles and different axes of symmetry. No such inclusion holds for thorns oriented in different ways and so the problem is much harder when the direction of the thorn is not fixed. Some preliminary estimates indicate that the problem is tractable using the same methods as in [1].

We will use many methods and ideas from [10], [12], [13], [15] and [19]. What we borrowed from those papers is the general structure of the proof - thorn points (times) are approximated by times when the path starts a relatively long sojourn inside the (translated) thorn, starting from a point strictly inside the thorn but close to its tip. The implementation of this idea for Brownian cone points (see, e.g., [10]) depended on estimates for the hitting time of the cone boundary. The discontinuity of stable paths requires some essentially new ideas. Informally, one has to slice the thorn $D(f)$ into pieces of the form $\left\{x \in D(f): x_{1} \in\left[2^{-k}, 2^{-k+1}\right)\right\}$. Then one has to estimate probabilities of various possible sequences of jumps of the stable process between the pieces of the thorn. The main estimates of this type are contained in Lemmas 3.2 and 4.5.

The paper has three more sections. The next section contains a review of wellknown results on stable processes which are needed in the proof of Theorem 1.1. Some of these results can be found in [3], a very well written monograph on Lévy processes. A very useful review of potential theoretic aspects of symmetric stable processes relevant to our project may be found in [6], [8] and [11]. Section 3 contains the proof of the "easy" part of Theorem 1.1, that is, part (i). Section 4 is the longest and most technical of all sections-it presents the proof of Theorem 1.1(ii).

2. Preliminaries. Let $\mathbf{N}=\{0,1,2, \ldots\}$ denote the set of natural numbers and $\mathbf{N}_{+}=\mathbf{N} \backslash\{0\}$. Let $d \in \mathbf{N}_{+}$. By $|\cdot|$ we will denote the Euclidean norm in $\mathbf{R}^{d}$. For any subset $U \subset \mathbf{R}^{d}$ we will denote its complement by $U^{c}$, its closure by $\bar{U}$ and its boundary by $\partial U$. Furthermore, for $x \in \mathbf{R}^{d}, r>0$ and $U, V \subset \mathbf{R}^{d}$ we put $B(x, r)=$ 
$\left\{y \in \mathbf{R}^{d}:|x-y|<r\right\}, r U=\{r y: y \in U\}, \operatorname{dist}(U, V)=\inf \{|y-z|: y \in U, z \in V\}$ and $\delta_{U}(x)=\operatorname{dist}(x, \partial U)$. We will write $m(U)$ for the $d$-dimensional Lebesgue measure of the set $U \subset \mathbf{R}^{d} . \mathscr{B}\left(\mathbf{R}^{d}\right)$ will denote the Borel $\sigma$-algebra of $\mathbf{R}^{d}$.

We will write $c=c(\alpha, \beta, \ldots, \gamma)$ to indicate the dependence of a constant $c$ on parameters, functions, etc. The constants may change their value from one use to the next, even on the same line in the same formula. However, the set of parameters on which a constant may depend will not change from its one use to another. The constants denoted with $c$ will be always assumed finite and strictly positive.

From now on let $\alpha \in(0,2)$ and $d \in \mathbf{N}, d \geq 2$, unless stated otherwise. We will follow terminology and notation from [4] most of the time.

We will assume that the probability space $\Omega$ on which the process $X(t)$ is defined is the "canonical" space of all functions $\omega$ from $[0, \infty)$ to $\mathbf{R}^{d}$ such that $t \rightarrow \omega(t)$ is right continuous and has left limits. $X(t, \omega)$ is given by $X(t, \omega)=$ $\omega(t)$. Shift operators will be denoted by $\theta_{t}(t \geq 0)$, that is, $\theta_{t}: \Omega \rightarrow \Omega,\left(\theta_{t} \omega\right)(s)=$ $\omega(t+s)$. The operators $\theta_{t}$ are also extended to stopping times $T$ and are denoted then by $\theta_{T}$. Since some of the stopping times may equal $\infty$ (for some $\omega \in \Omega$ ) it will be convenient to use the following convention (see [4] for more details). For each $\omega \in \Omega$ we put $X(\infty, \omega)=\omega(\infty)=\Delta$, where $\Delta$ is an extra point not in $\mathbf{R}^{d}$. We also add to $\Omega$ a point $\omega_{\Delta}$ such that for all $t \in[0, \infty]$ we have $X\left(t, \omega_{\Delta}\right)=\omega_{\Delta}(t)=\Delta$, $\theta_{\infty} \omega=\omega_{\Delta}, \omega \in \Omega$.

A symmetric $\alpha$-stable process $X(t)$ can be constructed in a formal way so that it is a standard process ([4], Chapter I, Definition 9.2) and satisfies the following properties. The distribution of the process starting from $x \in \mathbf{R}^{d}$ will be denoted by $P^{x}$, and $E^{x}$ will denote the corresponding expectation. We will assume that $\Omega$ is equipped with a $\sigma$-field $\mathcal{M}$ which is "universally complete" in the following sense: if $F \in \mathcal{M}$ and $P^{x}(F)=0$ for all $x \in \mathbf{R}^{d}$ then every subset of $F$ is in $\mathcal{M}$.

Let $F \in \mathcal{M}$ and $Y$ be an $\mathcal{M}$-measurable random variable. If we write $P(F)=a$ [or $E(Y)=a$ ], this will mean that $a$ is the common value of $P^{x}(F)$ for all $x \in \mathbf{R}^{d}$ [or $E^{x}(Y)$ respectively].

We will denote by $p(t, x, y)=p_{t}(x-y), t>0, x, y \in \mathbf{R}^{d}$, the transition densities of $X(t)$, that is, $P^{x}(X(t) \in U)=\int_{U} p(t, x, y) d y, U \in \mathscr{B}\left(\mathbf{R}^{d}\right)$. For each $t>0$ the function $p_{t}(\cdot)$ is bounded and continuous on $\mathbf{R}^{d}$ and has the following useful scaling property, $p_{t}(x)=t^{-d / \alpha} p_{1}\left(x / t^{1 / \alpha}\right), x \in \mathbf{R}^{d}$.

The Lévy measure of the process $X(t)$ is of the form $v(d x)=C_{d,-\alpha}|x|^{-\alpha-d} d x$ where

$$
C_{d, \gamma}=\Gamma((d-\gamma) / 2) /\left(2^{\gamma} \pi^{d / 2}|\Gamma(\gamma / 2)|\right) .
$$

For an open set $U \subset \mathbf{R}^{d}$ we define $\tau_{U}=\inf \left\{t \geq 0: X(t) \in U^{c}\right\}$, the first entry time of $U^{c}$. It is well known that $\tau_{a U}$ and $a^{\alpha} \tau_{U}(a>0)$ have the same distribution under $P^{0}$. In the sequel we will need the following elementary estimate. If $U$ is an open bounded set then

$$
P^{x}\left(\tau_{U}>t\right) \leq c_{1}(\alpha, U) \exp \left(-c_{2}(\alpha, U) t\right), \quad t \geq 0, x \in \mathbf{R}^{d} .
$$


We will briefly indicate how to prove (2.1). Since $p_{1}(x)$ is everywhere positive and $U$ is bounded, $P^{y}\left(\tau_{U}>1\right) \leq P^{y}(X(1) \in U) \leq c_{3}(\alpha, U)<1$ for all $y \in \mathbf{R}^{d}$. By the Markov property, $P^{y}\left(\tau_{U}>k\right) \leq c_{3}^{k}$ for all $k \geq 1$ and $y \in \mathbf{R}^{d}$, from which (2.1) follows.

$X^{(1)}(t), \ldots, X^{(d)}(t)$ will denote one-dimensional components of the process $X(t)$, that is, $X(t)=\left(X^{(1)}(t), \ldots, X^{(d)}(t)\right)$. It is known that each component $X^{(i)}(t)$ is a symmetric $\alpha$-stable process on the line. For any open set $U \subset \mathbf{R}$ and $i=1, \ldots, d$, we will denote

$$
\tau_{U}^{(i)}=\inf \left\{t \geq 0: X^{(i)}(t) \in U^{c}\right\} .
$$

For an open set $U \subset \mathbf{R}^{d}$ and $x \in \mathbf{R}^{d}$, the distribution $P^{x}\left(\tau_{U}<\infty ; X\left(\tau_{U}\right) \in \cdot\right)$ will be called the $\alpha$-harmonic measure for $U$ and denoted by $\mu_{U}^{x}(\cdot)$. It is well known that for any $a>0$ and $V \in \mathcal{B}\left(\mathbf{R}^{d}\right)$, the following scaling property holds:

$$
P^{a x}\left(X\left(\tau_{a U}\right) \in a V\right)=P^{x}\left(X\left(\tau_{U}\right) \in V\right) .
$$

In the case of a ball $B(0, r), r>0$, for any $x \in B(0, r)$, the $\alpha$-harmonic measure $\mu_{B(0, r)}^{x}$ has a density function $\mathcal{Q}_{r}(x, \cdot)$ (the Poisson kernel) given by the formula

$$
\mathcal{Q}_{r}(x, y)= \begin{cases}C_{\alpha}^{d}\left(r^{2}-|x|^{2}\right)^{\alpha / 2}\left(|y|^{2}-r^{2}\right)^{-\alpha / 2}|x-y|^{-d}, & \text { for }|y|>r \\ 0, & \text { for }|y| \leq r\end{cases}
$$

where $C_{\alpha}^{d}=\Gamma(d / 2) \pi^{-d / 2-1} \sin (\pi \alpha / 2)$ [5]. This formula holds also for $d=1$.

Similarly, in the case of the complement of the ball $\overline{B(0, r)}$, for any $x \in$ $(\overline{B(0, r)})^{c}$, the $\alpha$-harmonic measure $\mu^{x} \overline{(\overline{B(0, r)})^{c}}$ has a density

$$
\widehat{\mathcal{Q}}_{r}(x, y)= \begin{cases}C_{\alpha}^{d}\left(|x|^{2}-r^{2}\right)^{\alpha / 2}\left(r^{2}-|y|^{2}\right)^{-\alpha / 2}|x-y|^{-d}, & \text { for }|y|<r \\ 0, & \text { for }|y| \geq r .\end{cases}
$$

We would like to point out that this formula does not apply when $d=1$ and $\alpha>1$.

We can easily derive a formula for the density of the $\alpha$-harmonic measure for the half-space $\mathscr{H}=\left\{x \in \mathbf{R}^{d}: x_{1}<0\right\}$ using (2.3) and (2.4). Fix some $x=\left(x_{1}, \ldots, x_{d}\right) \in \mathscr{H}$. One can use (2.3) applied with $d=1$ to show that $P^{x_{1}}\left(X^{(1)}\left(\tau_{(-n, 0)}^{(1)}\right)=0\right)=0$ for all integer $n \geq 1$. This easily implies that $P^{x_{1}}\left(X^{(1)}\left(\tau_{(-\infty, 0)}^{(1)}\right)=0\right)=0$. Hence $P^{x}\left(X\left(\tau_{\mathscr{H}}\right) \in \partial \mathscr{H}\right)=0$. Next, for any $n \in \mathbf{N}_{+}$ let $v_{n}=\left(-n, x_{2}, \ldots, x_{d}\right), w_{n}=\left(n, x_{2}, \ldots, x_{d}\right), V(n)=B\left(v_{n}, n\right)$ and $W(n)=$ $\left(\overline{B\left(w_{n}, n\right)}\right)^{c}$. For any $U \subset(\overline{\mathscr{H}})^{c}, U \in \mathscr{B}\left(\mathbf{R}^{d}\right)$, we have

$$
\begin{aligned}
P^{x}\left(X\left(\tau_{W(n)}\right) \in U \cap(W(n))^{c}\right) & \geq P^{x}\left(X\left(\tau_{\mathscr{H}}\right) \in U \cap(W(n))^{c}\right) \\
& \geq P^{x}\left(X\left(\tau_{V(n)}\right) \in U \cap(W(n))^{c}\right) .
\end{aligned}
$$

These inequalities combined with (2.3) and (2.4) yield upon passing to the limit, when $n \rightarrow \infty$, the following formula for the density of the $\alpha$-harmonic measure $\mu_{\mathscr{H}}^{x}$ :

$$
Q_{\mathscr{H}}(x, y)= \begin{cases}C_{\alpha}^{d} \delta_{\mathscr{H}}^{\alpha / 2}(x) \delta_{\mathscr{H}}^{-\alpha / 2}(y)|x-y|^{-d}, & \text { for } y \in(\overline{\mathscr{H}})^{c}, \\ 0, & \text { for } y \in \overline{\mathscr{H}},\end{cases}
$$


for $x \in \mathscr{H}$.

We define the potential kernel $u(x, y)$ of the process $X(t)$ by

$$
u(x, y)=\int_{0}^{\infty} p(t, x, y) d t, \quad x, y \in \mathbf{R}^{d} .
$$

According to [4] we have $u(x, y)=C_{d, \alpha}|x-y|^{\alpha-d}$ (the Riesz kernel). For any open set $U \subset \mathbf{R}^{d}$ we define

$$
G_{U}(x, y)=u(x, y)-E^{x}\left(\tau_{U}<\infty ; u\left(X\left(\tau_{U}\right), y\right)\right), \quad x, y \in \mathbf{R}^{d}, x \neq y,
$$

and call $G_{U}(x, y)$ the Green function for $U$. We put $G_{U}(x, x)=\infty$ if $x \in U$ and $G_{U}(x, x)=0$ if $x \notin U$. It is well known that if $U_{1} \subset U_{2}$ are open sets then we have $G_{U_{1}}(x, y) \leq G_{U_{2}}(x, y), x, y \in \mathbf{R}^{d}$. For any open set $U$ and nonnegative Borel measurable function $h$, the Green function satisfies

$$
E^{x} \int_{0}^{\tau_{U}} h(X(t)) d t=\int_{U} G_{U}(x, y) h(y) d y, \quad x \in \mathbf{R}^{d} .
$$

In particular, we have

$$
E^{x}\left(\tau_{U}\right)=\int_{U} G_{U}(x, y) d y .
$$

Suppose that $U \subset \mathbf{R}^{d}$ is an open, nonempty, bounded set, $V \in \mathscr{B}\left(\mathbf{R}^{d}\right)$ and $\operatorname{dist}(U, V)>0$. The following formula recovers the $\alpha$-harmonic measure from the Green function. A connection between the Lévy measure and the harmonic measure established in [16] yields for $x \in U$,

$$
P^{x}\left(X\left(\tau_{U}\right) \in V\right)=\int_{U} G_{U}(x, y) \int_{V} \frac{C_{d,-\alpha}}{|y-z|^{d+\alpha}} d z d y .
$$

We can combine (2.7) and (2.8) to obtain

$$
P^{x}\left(X\left(\tau_{U}\right) \in V\right) \leq c(d, \alpha) E^{x}\left(\tau_{U}\right) \sup \left\{|y-z|^{-d-\alpha}: y \in U, z \in V\right\} m(V) .
$$

Using (2.8) we can express $\mathcal{Q}_{r}(x, z), r>0, x \in B(0, r), z \in \mathbf{R}^{d},|z|>r$, in terms of the Green function for $B(0, r)$, namely,

$$
\mathcal{Q}_{r}(x, z)=\int_{B(0, r)} G_{B(0, r)}(x, y) \frac{C_{d,-\alpha}}{|y-z|^{d+\alpha}} d y .
$$

Recall (2.3), multiply both sides of the last formula by $|z|^{d+\alpha}$ and let $|z| \rightarrow \infty$ to see that

$$
\begin{aligned}
E^{x} \tau_{B(0, r)} & =\int_{B(0, r)} G_{B(0, r)}(x, y) d y \\
& =C_{\alpha}^{d}\left(C_{d,-\alpha}\right)^{-1}\left(r^{2}-|x|^{2}\right)^{\alpha / 2}, \quad|x|<r .
\end{aligned}
$$

This formula holds also for $d=1$. 
It will be convenient to use a summability condition for a series in place of the integral criterion given in Theorem 1.1. Let

$$
J(f)=\sum_{k=1}^{\infty} \frac{\left(f\left(2^{-k}\right)\right)^{d+\alpha-1}}{\left(2^{-k}\right)^{d+\alpha-1}} .
$$

We leave the proof of the following elementary result to the reader.

LEMMA 2.1. $I(f)<\infty$ if and only if $J(f)<\infty$.

3. Proof of Theorem 1.1(i). Recall that $t_{0}>0$ is fixed and assume that $I(f)=\infty$. Let $g(x)=f(x) \wedge x$. It is elementary to see that $I(f)=\infty$ implies that $I(g)=\infty$. To prove Theorem 1.1(i), it will suffice to show that $P(A(g))=1$.

We need some more notation. Let $\left\{a_{n}\right\}_{n=1}^{\infty}$ be a sequence of natural numbers satisfying $a_{1}=2, a_{n+1}>a_{n}, n \in \mathbf{N}_{+}$. The values of $a_{n}$ 's will be chosen later in this section. For $n \in \mathbf{N}_{+}$we denote

$$
\begin{aligned}
& W(n)=\left\{x \in D(g): x_{1} \in\left(0,2^{-a_{n}}\right)\right\}, \\
& V(n)=\left\{x \in D(g): x_{1} \in\left[2^{-a_{n+1}}, 2^{-a_{n}}\right)\right\} .
\end{aligned}
$$

Additionally, we put

$$
\begin{aligned}
& W(0)=W(1) \cup\left\{x \in \mathbf{R}^{d}: x_{1} \in(1 / 2,1),|\tilde{x}|<g(1 / 2)\right\}, \\
& V(0)=\left\{x \in \mathbf{R}^{d}: x_{1} \in(4 / 6,5 / 6),|\tilde{x}|<g(1 / 2) / 2\right\} .
\end{aligned}
$$

For $n \in \mathbf{N}$ we denote

$$
U(n)=\left\{x \in \mathbf{R}^{d}: x_{1} \in\left[2^{-n-1}, 2^{-n}\right),|\tilde{x}|<g\left(2^{-n-2}\right) / 2\right\} .
$$

LEMMA 3.1. (i) For all $x \in U(n), n \in \mathbf{N}, n \geq 3$, we have

$$
P^{x}\left(X\left(\tau_{W(1)}\right) \in V(0)\right) \geq c(\alpha, d) g^{\alpha}\left(2^{-n-2}\right) \text {. }
$$

(ii) For all $x \in W(n), n \in \mathbf{N}_{+}$, and $k \leq a_{n}-2, k \in \mathbf{N}$, we have

$$
P^{x}\left(X\left(\tau_{W(n)}\right) \in U(k)\right) \geq c(\alpha, d) E^{x}\left(\tau_{W(n)}\right) g^{d-1}\left(2^{-k-2}\right)\left(2^{-k-2}\right)^{-d-\alpha+1} .
$$

PROOF. For each $x \in U(n)$ we have $B=B\left(x, g\left(2^{-n-2}\right) / 2\right) \subset W(1)$, for $n \geq 3$. Hence $P^{x}\left(X\left(\tau_{W(1)}\right) \in V(0)\right) \geq P^{x}\left(X\left(\tau_{B}\right) \in V(0)\right)$. By (2.8) the last expression is greater than or equal to

$$
c(\alpha, d) \int_{B} G_{B}(x, y) d y=c(\alpha, d) E^{x}\left(\tau_{B}\right)=c(\alpha, d) 2^{-\alpha} g^{\alpha}\left(2^{-n-2}\right) E^{0}\left(\tau_{B(0,1)}\right) .
$$

This proves the first part of the lemma.

Using (2.8) again we obtain

$$
\begin{aligned}
& P^{x}\left(X\left(\tau_{W(n)}\right) \in U(k)\right) \\
& \quad \geq c(\alpha, d) E^{x}\left(\tau_{W(n)}\right)(\sup \{|y-z|: y \in W(n), z \in U(k)\})^{-d-\alpha} m(U(k)) .
\end{aligned}
$$


Note that

$$
\sup \{|y-z|: y \in W(n), z \in U(k)\} \leq 2^{-k+1}
$$

and

$$
m(U(k))=c(\alpha, d) 2^{-k-2} g^{d-1}\left(2^{-k-2}\right) .
$$

Part (ii) of the lemma follows from the three formulae.

Consider the following events:

$$
\begin{aligned}
F_{1} & =\left\{X\left(\tau_{W(1)}\right) \in V(0)\right\}, \\
F_{n+1} & =\left\{X\left(\tau_{W(n+1)}\right) \in V(n)\right\} \cap \theta_{\tau_{W(n+1)}^{-1}} F_{n}, \quad n \in \mathbf{N}_{+} .
\end{aligned}
$$

Note that on the set $\left\{X\left(\tau_{W(n+1)}\right) \in V(n)\right\}$ we have

$$
\theta_{\tau_{W(n+1)}^{-1}}^{-1}\left\{X\left(\tau_{W(k)}\right) \in V(k-1)\right\}=\left\{X\left(\tau_{W(k)}\right) \in V(k-1)\right\}
$$

for any $n \geq k \geq 1, k, n \in \mathbf{N}$, so that $F_{n+1}=\bigcap_{k=1}^{n+1}\left\{X\left(\tau_{W(k)}\right) \in V(k-1)\right\}$.

Recall that $\left\{a_{n}\right\}$ is a sequence of natural numbers satisfying $a_{1}=2, a_{n+1}>a_{n}$, $n \in \mathbf{N}_{+}$.

LEMMA 3.2. One can find a sequence $\left\{a_{n}\right\}_{n=1}^{\infty}$ depending on $\alpha, d$ and $g$ such that, for all $n \in \mathbf{N}_{+}$and $x \in W(n)$,

$$
P^{x}\left(F_{n}\right) \geq \frac{1}{2} P^{x}\left(F_{1}\right) .
$$

PROOF. Let $H_{n}=F_{1} \backslash F_{n}$ and $c_{n}=n /(n+1), n \in \mathbf{N}_{+}$. We will show that there exists a sequence $\left\{a_{n}\right\}_{n=1}^{\infty}$ such that

$$
P^{x}\left(H_{n}\right) \leq c_{n} P^{x}\left(F_{n}\right), \quad x \in W(n), n \in \mathbf{N}_{+} .
$$

Clearly (3.1) follows from (3.2).

We will prove (3.2) by induction. For $n=1,(3.2)$ is trivial. Assume that we have chosen $a_{1}, a_{2}, \ldots, a_{m}$ for some $m \in \mathbf{N}_{+}$and that (3.2) holds for $n=1,2, \ldots, m$. Consider some $a_{m+1} \in \mathbf{N}$ with $a_{m+1}>a_{m}$, whose value will be chosen later in the proof.

For any $x \in W(m+1)$ we have

$$
P^{x}\left(F_{m+1}\right) \geq \sum_{k=a_{m} \vee 3}^{a_{m+1}-2} E^{x}\left(X\left(\tau_{W(m+1)}\right) \in U(k) ; P^{X\left(\tau_{W(m+1)}\right)}\left(F_{m}\right)\right) .
$$

For $z \in U(k)$, where $k \in \mathbf{N}, a_{m} \vee 3 \leq k \leq a_{m+1}-2$, we obtain from (3.2) and Lemma 3.1(i),

$$
P^{z}\left(F_{m}\right) \geq \frac{1}{2} P^{z}\left(F_{1}\right) \geq c(\alpha, d) g^{\alpha}\left(2^{-k-2}\right)
$$


From this and Lemma 3.1(ii) we get

$$
P^{x}\left(F_{m+1}\right) \geq c(\alpha, d) E^{x}\left(\tau_{W(m+1)}\right) \sum_{k=a_{m} \vee 3}^{a_{m+1}-2} \frac{\left(g\left(2^{-k-2}\right)\right)^{d+\alpha-1}}{\left(2^{-k-2}\right)^{d+\alpha-1}},
$$

$$
x \in W(m+1) .
$$

On the other hand, for any $x \in W(m+1)$, we have

$$
\begin{aligned}
P^{x}\left(H_{m+1}\right)= & P^{x}\left(F_{1} ; X\left(\tau_{W(m+1)}\right) \in V(m) ;\left(\theta_{\tau_{W(m+1)}}^{-1} F_{m}\right)^{c}\right) \\
& +P^{x}\left(F_{1} ; X\left(\tau_{W(m+1)}\right) \notin V(m)\right) \\
= & I+I I .
\end{aligned}
$$

Applying (3.2) with $n=m$ we obtain

$$
\begin{aligned}
I & =E^{x}\left(X\left(\tau_{W(m+1)}\right) \in V(m) ; P^{X\left(\tau_{W(m+1)}\right)}\left(F_{m}^{c} \cap F_{1}\right)\right) \\
& \leq c_{m} E^{x}\left(X\left(\tau_{W(m+1)}\right) \in V(m) ; P^{X\left(\tau_{W(m+1)}\right)}\left(F_{m}\right)\right)=c_{m} P^{x}\left(F_{m+1}\right) .
\end{aligned}
$$

Using (2.9) we get

$$
\begin{aligned}
I I & \leq P^{x}\left(X\left(\tau_{W(m+1)}\right) \in W(0) \backslash V(m)\right) \\
& \leq c(\alpha, d)\left(2^{-a_{m}}-2^{-a_{m+1}}\right)^{-\alpha-d} E^{x}\left(\tau_{W(m+1)}\right) \\
& \leq c(\alpha, d)\left(2^{-a_{m}}\right)^{-\alpha-d} E^{x}\left(\tau_{W(m+1)}\right) .
\end{aligned}
$$

We have $J(g)=\infty$. Hence, in view of (3.3) we can take $a_{m+1}$ large enough so that $I I \leq\left(c_{m+1}-c_{m}\right) P^{x}\left(F_{m+1}\right), x \in W(m+1)$. This, (3.5) and (3.4) give (3.2) for $n=m+1$. This completes the proof of the induction step.

For the remainder of this section, let $\left\{a_{n}\right\}_{n=1}^{\infty}$ be a fixed sequence chosen as in Lemma 3.2. For each $n \in \mathbf{N}_{+}$fix a point $y_{n} \in V(n) \cap\left\{x \in \mathbf{R}^{d}:|\tilde{x}|=0\right\}$.

For $n \in \mathbf{N}_{+}$and $k=1,2, \ldots, n$ consider the following stopping times:

$$
S_{k}^{n}=\inf \left\{t \geq 0: X(t) \notin X(0)-y_{n}+W(n-k+1)\right\} .
$$

We will define another sequence of stopping times $R_{n}$. We let $R_{n}$ be the first of these times $S_{1}^{n}, S_{2}^{n}, \ldots, S_{n}^{n}$ for which $X\left(S_{k}^{n}\right) \notin X(0)-y_{n}+V(n-k)$. If there is no such $S_{k}^{n}$, then we put $R_{n}=\inf \left\{t \geq 0: X(t) \notin X(0)-y_{n}+W(0)\right\}$.

LEMMA 3.3. There exists a constant $c=c\left(\alpha, d, g, t_{0}\right)$ such that

$$
P\left(R_{n} \geq t_{0}\right) \geq c E\left(R_{n}\right), \quad n \in \mathbf{N}_{+} .
$$


ProOF. We have

$$
\begin{aligned}
E\left(R_{n}\right) & =E^{y_{n}}\left(R_{n}\right) \\
& \leq E^{y_{n}}\left(\tau_{W(0)}\right) \\
& =E^{y_{n}}\left(\tau_{W(1)}\right)+E^{y_{n}}\left(X\left(\tau_{W(1)}\right) \in W(0) \backslash W(1) ; E^{X\left(\tau_{W(1)}\right)}\left(\tau_{W(0)}\right)\right) .
\end{aligned}
$$

The last term can be bounded by $c(\alpha, d) E^{y_{n}}\left(\tau_{W(1)}\right)$ using (2.9). Hence,

$$
E\left(R_{n}\right) \leq c(\alpha, d) E^{y_{n}}\left(\tau_{W(1)}\right) .
$$

On the other hand, we have

$$
\begin{aligned}
P\left(R_{n} \geq t_{0}\right) & =P^{y_{n}}\left(R_{n} \geq t_{0}\right) \\
& \geq P^{y_{n}}\left(\bigcap_{j=1}^{n}\left\{X\left(\tau_{W(j)}\right) \in V(j-1)\right\} \cap\left\{\tau_{W(0)} \circ \theta_{\tau_{W(1)}} \geq t_{0}\right\}\right) .
\end{aligned}
$$

This is equal to

$$
E^{y_{n}}\left(F_{n} ; X\left(\tau_{W(1)}\right) \in V(0) ; P^{X\left(\tau_{W(1)}\right)}\left(\tau_{W(0)} \geq t_{0}\right)\right) .
$$

Note that $\inf _{x \in V(0)} P^{x}\left(\tau_{W(0)} \geq t_{0}\right)$ is strictly positive so the last expression is greater than $c\left(\alpha, d, g, t_{0}\right) P^{y_{n}}\left(F_{n}\right)$. By Lemma 3.2 and (2.8), this is greater than $c E^{y_{n}}\left(\tau_{W(1)}\right)$. We have shown that $P\left(R_{n} \geq t_{0}\right) \geq c E^{y_{n}}\left(\tau_{W(1)}\right)$. The argument can be completed by combining this inequality with an upper bound for $E R_{n}$ obtained earlier in the proof.

For $j, l \in \mathbf{N}$ and $n \in \mathbf{N}_{+}$let us define the following stopping times:

$$
\begin{aligned}
T(0, n, l) & =2 l t_{0}, \\
T(j+1, n, l) & = \begin{cases}T(j, n, l)+\left(R_{n} \wedge t_{0}\right) \circ \theta_{T(j, n, l)}, & \text { if } T(j, n, l)<2 l t_{0}+t_{0}, \\
T(j, n, l), & \text { if } T(j, n, l) \geq 2 l t_{0}+t_{0} .\end{cases}
\end{aligned}
$$

Next, for $j, n, l$ as above define events

$$
F(j, n, l)=\left\{T(j+1, n, l)-T(j, n, l)=t_{0}\right\} .
$$

Let us also put $H_{n}^{l}=\bigcup_{j=0}^{\infty} F(j, n, l), l \in \mathbf{N}, n \in \mathbf{N}_{+}$.

The proof of the following lemma is based on ideas from [12], Section 2, and [10].

LEMMA 3.4. There exists $n_{0}=n_{0}\left(\alpha, d, t_{0}\right) \in \mathbf{N}_{+}$such that, for all $n \geq n_{0}$, $n \in \mathbf{N}$, and all $l \in \mathbf{N}$, we have $P\left(H_{n}^{l}\right) \geq c\left(\alpha, d, g, t_{0}\right)$.

Proof. First note that $E\left(R_{n}\right) \rightarrow 0$ as $n \rightarrow \infty$. Indeed, $E^{y_{n}}\left(R_{n}\right) \leq E^{y_{n}}\left(\tau_{U}\right)$, where $U=\left\{x \in \mathbf{R}^{d}: x_{1} \in(0,1)\right\}$. But $E^{y_{n}}\left(\tau_{U}\right)$ equals $c(\alpha, d)\left((1 / 2)^{\overline{2}}-\mid 1 / 2-\right.$ $\left.\left.\left|y_{n}\right|\right|^{2}\right)^{\alpha / 2}$, by (2.10). 
Hence, for all $n \geq n_{0}\left(\alpha, d, t_{0}\right) \in \mathbf{N}_{+}, n \in \mathbf{N}$, there exists $m_{n} \in \mathbf{N}$ such that

$$
t_{0} / 3 \leq m_{n} E\left(R_{n}\right) \leq t_{0} / 2 .
$$

It follows that

$$
E\left(T\left(m_{n}, n, l\right)-2 l t_{0}\right)=E\left(\sum_{j=1}^{m_{n}}[T(j, n, l)-T(j-1, n, l)]\right) \leq m_{n} E\left(R_{n}\right) \leq t_{0} / 2 .
$$

Hence

$$
P\left(T\left(m_{n}, n, l\right)-2 l t_{0} \geq t_{0}\right) \leq 1 / 2 .
$$

Since $F\left(j_{1}, n, l\right) \cap F\left(j_{2}, n, l\right)=\varnothing$ for $j_{1} \neq j_{2}$,

$$
\begin{aligned}
P\left(H_{n}^{l}\right) & \geq P\left(\bigcup_{j=1}^{m_{n}} F(j, n, l)\right) \\
& =\sum_{j=1}^{m_{n}} P(F(j, n, l)) \\
& =\sum_{j=1}^{m_{n}} P\left(T(j, n, l)<2 l t_{0}+t_{0}\right) P\left(R_{n} \geq t_{0}\right) \\
& \geq m_{n} P\left(T\left(m_{n}, n, l\right)<2 l t_{0}+t_{0}\right) P\left(R_{n} \geq t_{0}\right) .
\end{aligned}
$$

By Lemma 3.3 and (3.7) this is bounded from below by $c\left(\alpha, d, g, t_{0}\right) m_{n} E\left(R_{n}\right)$. This proves the lemma in view of (3.6).

Proof OF TheOREM 1.1(i). For any $l \in \mathbf{N}$ let $H^{l}=\lim _{n \rightarrow \infty} \sup _{n \rightarrow \infty} H_{n}$ and define the following subset of $\Omega$ :

$$
\begin{aligned}
A^{l}=\{\omega \in \Omega: & \exists s=s(\omega) \in\left[2 l t_{0}, 2 l t_{0}+t_{0}\right] \text { such that } \\
& \left.X(t, \omega) \in X(s, \omega)+\bar{D}(g) \text { for all } t \in\left[s, s+t_{0}\right)\right\} .
\end{aligned}
$$

We will show that $H^{l} \subset A^{l}$ for all $l \in \mathbf{N}$. Assume that $\omega \in H^{l}$. Then there exist strictly increasing sequences $\left\{j_{k}\right\}_{k=1}^{\infty}$ and $\left\{n_{k}\right\}_{k=1}^{\infty}$ (both depending on $\omega$ ) such that $\omega \in \bigcap_{k=1}^{\infty} F\left(j_{k}, n_{k}, l\right)$. We may assume by passing to a subsequence, if necessary, that the sequence $T\left(j_{k}, n_{k}, l, \omega\right)$ is convergent to $s$ as $k \rightarrow \infty$ for some $s=s(\omega) \in\left[2 l t_{0}, 2 l t_{0}+t_{0}\right]$. We can also assume that $\left\{T\left(j_{k}, n_{k}, l, \omega\right)\right\}_{k=1}^{\infty}$ is either strictly increasing or nonincreasing. We will write $s_{k}=T\left(j_{k}, n_{k}, l, \omega\right)$ for short.

First consider the case when this sequence is strictly increasing to $s$. Recall that all paths have left-hand limits and are right continuous. We will argue that the path $t \rightarrow X(t, \omega)$ is continuous at $s$, that is, $\lim _{t \rightarrow s} X(t, \omega)=X(s, \omega)$. 
If $\omega \in F\left(j_{k}, n_{k}, l\right)$ then, for $t \in\left[s_{k}, s_{k}+t_{0}\right)$, the path $X(t, \omega)$ stays in $X\left(s_{k}, \omega\right)-$ $y_{n_{k}}+W(0)$. Moreover, for $t \in\left[s_{k}, s_{k}+t_{0}\right)$ and each $m=0,1, \ldots, n_{k}-1$, if the path $X(t, \omega)$ leaves $X\left(s_{k}, \omega\right)-y_{n_{k}}+W\left(n_{k}-m\right)$ then it goes to $X\left(s_{k}, \omega\right)-y_{n_{k}}+$ $V\left(n_{k}-m-1\right)$. Note also that $X\left(s_{k}, \omega\right)-y_{n_{k}}$ tends to $\lim _{t \uparrow s} X(t, \omega)$ as $k \rightarrow \infty$.

Suppose that $X(t, \omega)$ has a jump at $t=s$, that is $\lim _{t \uparrow s} X(t, \omega) \neq X(s, \omega)$. Then for large $k$, since $s_{k} \uparrow s$, the path $X(t, \omega)$ would have to jump at time $t=s$ from one of the sets $X\left(s_{k}, \omega\right)-y_{n_{k}}+W\left(n_{k}-m\right)$ to a point in the complement of $X\left(s_{k}, \omega\right)-y_{n_{k}}+V\left(n_{k}-m-1\right)$, for some $m \in\left\{0,1, \ldots, n_{k}-1\right\}$. This contradiction shows that $X(t, \omega)$ is continuous at $t=s$ if $\left\{s_{k}\right\}_{k=1}^{\infty}$ is strictly increasing. Thus $\lim _{k \rightarrow \infty} X\left(s_{k}, \omega\right)=X(s, \omega)$ in this case.

The same holds if $\left\{s_{k}\right\}_{k=1}^{\infty}$ is nonincreasing, by the right continuity of the trajectories.

In both cases we have

$X(t, \omega) \in X\left(s_{k}, \omega\right)-y_{n_{k}}+W(0) \subset X\left(s_{k}, \omega\right)-y_{n_{k}}+D(g) \quad$ for $t \in\left[s_{k}, s_{k}+t_{0}\right)$.

Since $\lim _{k \rightarrow \infty} X\left(s_{k}, \omega\right)=X(s, \omega)$ and $\lim _{k \rightarrow \infty} y_{n_{k}}=0$, we conclude that $X(t, \omega) \in X(s, \omega)+\bar{D}(g)$ for all $t \in\left[s, s+t_{0}\right)$. This completes the proof of $H^{l} \subset A^{l}$.

Note that $(A(g))^{c} \subset \bigcap_{l=0}^{\infty}\left(A^{l}\right)^{c} \subset \bigcap_{l=0}^{\infty}\left(H^{l}\right)^{c}$. By Lemma 3.4, we have $P\left(H^{l}\right) \geq c\left(\alpha, d, g, t_{0}\right)$. The events $\left\{\left(H^{l}\right)^{c}\right\}_{l=0}^{\infty}$ are independent so $P\left(\bigcap_{l=0}^{\infty}\left(H^{l}\right)^{c}\right)=0$. Hence, we obtain $P(A(g))=1$.

4. Proof of Theorem 1.1(ii). We will start by deriving some estimates for the $\alpha$-harmonic measure of a $d$-dimensional cylinder. The estimates in Lemmas 4.2 and 4.3 are more or less standard. Similar estimates were obtained in [9], Lemma 3. However, since we could not find a direct reference for our estimates, we will spell out their proofs in detail.

We put

$$
\mathcal{C}(r)=\left\{x \in \mathbf{R}^{d}: x_{1}<0,|\tilde{x}|<r\right\}, \quad r>0 .
$$

The "unit" cylinder $\mathcal{C}(1)$ will be denoted $\mathcal{C}$ for short. Let $\Phi(a, b)=\left\{x \in \mathbf{R}^{d}: a<\right.$ $\left.x_{1}<b,|\tilde{x}|<1\right\}$, and similarly, $\Phi[a, b)=\left\{x \in \mathbf{R}^{d}: a \leq x_{1}<b,|\tilde{x}|<1\right\}$. We will write $\mathscr{H}=\left\{x \in \mathbf{R}^{d}: x_{1}<0\right\}$.

Our next lemma says that although $\mathcal{C}$ is not bounded, (2.8) holds for $\mathcal{C}$ in place of $U$.

LEMMA 4.1. Let $V \subset \mathscr{H}^{c}$ be a Borel set such that $\operatorname{dist}(\mathcal{C}, V)>0$. For any $x \in \mathcal{C}$ we have

$$
\begin{aligned}
P^{x}\left(X\left(\tau_{\mathcal{C}}\right) \in V\right) & =\int_{\mathcal{C}} G_{\mathcal{C}}(x, y) \int_{V} \frac{C_{d,-\alpha}}{|y-z|^{d+\alpha}} d z d y \\
& \leq c(\alpha, d) E^{x}\left(\tau_{\mathcal{C}}\right) \sup \left\{|y-z|^{-d-\alpha}: y \in \mathcal{C}, z \in V\right\} m(V) .
\end{aligned}
$$

We also have $E^{x}\left(\tau_{\mathcal{C}}\right) \leq c(\alpha), x \in \mathcal{C}$. 
ProOF. Recall (2.2), put $F_{n}=\left(X^{(1)}\left(\tau_{(-n, 0)}^{(1)}\right) \in(-\infty,-n]\right), n \in \mathbf{N}_{+}$, and note that $F_{n+1} \subset F_{n}, n \in \mathbf{N}_{+}$. Fix any $x \in \mathcal{C}$. By (2.3) applied with $d=1$ we have $\lim _{n \rightarrow \infty} P^{x}\left(F_{n}\right)=0$.

Therefore for $P^{x}$-almost all $\omega \in \Omega$ there exists $N=N(\omega) \in \mathbf{N}$ such that for all $n \geq N, n \in \mathbf{N}$, we have $\omega \notin F_{n}$ and consequently $\tau_{\Phi(-n, 0)}(\omega)=\tau_{\mathcal{C}}(\omega)$. It follows from (2.6) that $G_{\Phi(-n, 0)}(x, y)$ increases to $G_{\mathcal{C}}(x, y), y \in \mathbf{R}^{d}$ as $n \uparrow \infty$. Consequently, by letting $n \uparrow \infty$ in the formula (2.8) for $\Phi(-n, 0)$ and $V$ we get (4.1).

The last assertion of the lemma follows from the inequality $E^{x}\left(\tau_{\mathcal{C}}\right) \leq$ $E^{x_{2}}\left(\tau_{(-1,1)}^{(2)}\right)$.

LEMMA 4.2. For any $x=\left(x_{1}, \ldots, x_{d}\right) \in \mathcal{C}$ we have

$$
P^{x}\left(X\left(\tau_{\mathcal{C}}\right) \in \Phi\left[0,\left|x_{1}\right|\right)\right) \leq c(\alpha, d)\left|x_{1}\right|^{-d-\alpha+1} .
$$

PROOF. Let $l=\left|x_{1}\right| / 5$. By taking $c(\alpha, d)$ large enough we may and will assume that $l \geq 1$. We have

$$
\begin{aligned}
& P^{x}\left(X\left(\tau_{\mathcal{C}}\right) \in \Phi\left[0,\left|x_{1}\right|\right)\right) \\
& \leq P^{x}\left(X\left(\tau_{\Phi(-\infty,-4 l)}\right) \in \Phi[-3 l, 5 l)\right) \\
& +P^{x}\left(X\left(\tau_{\Phi(-\infty,-2 l)}\right) \in \Phi[-l, 5 l)\right) \\
& +P^{x}\left(X\left(\tau_{\Phi(-\infty,-4 l)}\right) \in \Phi[-4 l,-3 l) ;\right. \\
& \left.\quad X\left(\tau_{\Phi(-\infty,-2 l)}\right) \in \Phi[-2 l,-l) ; X\left(\tau_{\Phi(-\infty, 0)}\right) \in \Phi[0,5 l)\right) \\
& =I+I I+I I I .
\end{aligned}
$$

The three terms on the right-hand side represent three possible ways in which the process can go from $\mathcal{C}$ to $\Phi\left[0,\left|x_{1}\right|\right)$. The first two terms represent direct jumps while the last term corresponds to three jumps. Applying (4.1) we get $I+I I \leq c(\alpha, d) l^{-d-\alpha+1}$. By the strong Markov property we obtain

$$
\begin{aligned}
I I I=E^{x}\left[X\left(\tau_{\Phi(-\infty,-4 l)}\right) \in \Phi[-4 l,-3 l) ;\right. \\
E^{X\left(\tau_{\Phi(-\infty,-4 l)}\right)}\left[X\left(\tau_{\Phi(-\infty,-2 l)}\right) \in \Phi[-2 l,-l) ;\right. \\
\left.\left.P^{X\left(\tau_{\Phi(-\infty,-2 l)}\right)}\left(X\left(\tau_{\Phi(-\infty, 0)}\right) \in \Phi[0,5 l)\right)\right]\right] .
\end{aligned}
$$

Note that for any $y \in \Phi[-2 l,-l)$ we get, by $(2.5)$,

$$
\begin{aligned}
P^{y}\left(X\left(\tau_{\Phi(-\infty, 0)}\right) \in \Phi[0,5 l)\right) & \leq P^{y}\left(X\left(\tau_{\mathcal{H}}\right) \in \Phi[0,5 l)\right) \\
& \leq c(\alpha, d) l^{\alpha / 2-d} \int_{\Phi[0,5 l)} \delta_{\mathcal{H}}^{-\alpha / 2}(z) d z \\
& \leq c l^{-d+1} \leq c l^{(-d-\alpha+1) / 3} .
\end{aligned}
$$


Similarly, for $y \in \Phi[-4 l,-3 l)$, putting $y^{\prime}=y+(2 l, 0, \ldots, 0)$ and using translation invariance of $X$ we obtain

$$
P^{y}\left(X\left(\tau_{\Phi(-\infty,-2 l)}\right) \in \Phi[-2 l,-l)\right) \leq P^{y^{\prime}}\left(X\left(\tau_{\mathscr{H}}\right) \in \Phi[0, l)\right) \leq c l^{(-d-\alpha+1) / 3} .
$$

For the same reason,

$$
P^{x}\left(X\left(\tau_{\Phi(-\infty,-4 l)}\right) \in \Phi[-4 l,-3 l)\right) \leq c l^{(-d-\alpha+1) / 3} .
$$

We can combine the last three estimates to show that $I I I \leq c(\alpha, d) l^{-d-\alpha+1}$. This and similar estimates for $I$ and $I I$ prove the lemma.

LEMMA 4.3. Let $V \subset \mathscr{H}^{c}$ be an arbitrary Borel set, $r>0$ and $x=$ $\left(x_{1}, \ldots, x_{d}\right) \in \mathcal{C}(r)$ be such that $x_{1} \leq-r$. We have

$$
P^{x}\left(X\left(\tau_{\mathcal{C}(r)}\right) \in V\right) \leq c(\alpha, d) \int_{V} \frac{r^{\alpha}}{|x-z|^{d+\alpha}}\left(\frac{r^{\alpha / 2}}{\delta_{\mathcal{H}}^{\alpha / 2}(z)} \vee 1\right) d z
$$

Proof. By scaling we have $P^{x}\left(X\left(\tau_{\mathcal{C}(r)}\right) \in V\right)=P^{x / r}\left(X\left(\tau_{\mathcal{C}(1)}\right) \in r^{-1} V\right)$. The right-hand side of (4.2) has a similar scaling property so we may and will assume that $r=1$.

Let $V_{1}=\{z \in V: 3|x|<|z-x|\}$ and $V_{2}=V \backslash V_{1}$. For $z \in V_{1}$ we have $|z|>2|x|$, $|z-x|<2|z|$ and $\delta_{\mathcal{C}}(z) \geq|z| / 2$. Clearly, $\operatorname{dist}\left(\mathcal{C}, V_{1}\right)>0$. Throughout the proof $R$ will denote the integral on the right-hand side of (4.2). By (4.1) for $\mathcal{C}$ and $V_{1}$ we obtain

$$
\begin{aligned}
P^{x}\left(X\left(\tau_{\mathcal{C}}\right) \in V_{1}\right) & \leq c(\alpha, d) E^{x}\left(\tau_{\mathcal{C}}\right) \int_{V_{1}}|z|^{-d-\alpha} d z \\
& \leq c(\alpha, d) \int_{V_{1}}(|z-x| / 2)^{-d-\alpha} d z \leq c R
\end{aligned}
$$

Next we will estimate $P^{x}\left(X\left(\tau_{\mathcal{C}}\right) \in V_{2}\right)$. Our argument will be similar to that in the proof of Lemma 4.2. We will bound the probability in question by the sum of three terms which represent different ways in which the process can jump from $\mathcal{C}$ to $V_{2}$. Let $l=\left|x_{1}\right| / 3$. We have

$$
\begin{aligned}
& P^{x}\left(X\left(\tau_{\mathcal{C}}\right) \in V_{2}\right) \\
& \leq P^{x}\left(X\left(\tau_{\Phi(-\infty,-2 l)}\right) \in V_{2}\right) \\
&+E^{x}\left(X\left(\tau_{\Phi(-\infty,-2 l)}\right) \in \Phi[-l, 0) ; P^{X\left(\tau_{\Phi(-\infty,-2 l)}\right)}\left(X\left(\tau_{\mathcal{H}}\right) \in V_{2}\right)\right) \\
&+E^{x}\left(X\left(\tau_{\Phi(-\infty,-2 l)}\right) \in \Phi[-2 l,-l) ; P^{X\left(\tau_{\Phi(-\infty,-2 l)}\right)}\left(X\left(\tau_{\mathcal{H}}\right) \in V_{2}\right)\right) \\
&= I+I I+I I I .
\end{aligned}
$$

To complete the proof it will suffice to show that each of the three terms on the right-hand side is bounded by $c R$. The inequality $I \leq c R$ follows easily from (4.1). 
By (4.1) applied with $\Phi(-\infty,-2 l)$ and $\Phi[-l, 0)$ in place of $\mathcal{C}$ and $V$, and using (2.5),

$$
\begin{aligned}
I I & =\int_{\Phi(-\infty,-2 l)} G_{\Phi(-\infty,-2 l)}(x, v) \int_{\Phi[-l, 0)} \frac{C_{d,-\alpha}}{|v-y|^{d+\alpha}} P^{y}\left(X\left(\tau_{\mathcal{H}}\right) \in V_{2}\right) d y d v \\
& \leq \frac{c(\alpha, d)}{l^{d+\alpha}} \int_{\Phi(-\infty,-2 l)} G_{\Phi(-\infty,-2 l)}(x, v) d v \int_{\Phi[-l, 0)} P^{y}\left(X\left(\tau_{\mathcal{H}}\right) \in V_{2}\right) d y \\
& =\frac{c}{l^{d+\alpha}} E^{x} \tau_{\Phi(-\infty,-2 l)} \int_{\Phi[-l, 0)} P^{y}\left(X\left(\tau_{\mathcal{H}}\right) \in V_{2}\right) d y \\
& \leq \frac{c}{l^{d+\alpha}} \int_{\Phi[-l, 0)} P^{y}\left(X\left(\tau_{\mathscr{H}}\right) \in V_{2}\right) d y \\
& \leq \frac{c}{l^{d+\alpha}} \int_{\Phi[-l, 0)} \int_{V_{2}} \frac{\delta_{\mathscr{H}}^{\alpha / 2}(y)}{|y-z|^{d} \delta_{\mathscr{H}}^{\alpha / 2}(z)} d z d y .
\end{aligned}
$$

For $z \in V_{2}$ we have

$$
\int_{\Phi[-l, 0)} \frac{\delta_{\mathscr{H}}^{\alpha / 2}(y)}{|y-z|^{d}} d y \leq \int_{\Phi[-l, 0)}|y-z|^{\alpha / 2-d} d y \leq c(\alpha, d),
$$

so

$$
I I \leq \frac{c(\alpha, d)}{l^{d+\alpha}} \int_{V_{2}} \frac{1}{\delta_{\mathscr{H}}^{\alpha / 2}(z)} d z \leq c(\alpha, d) \int_{V_{2}} \frac{1}{|x-z|^{d+\alpha} \delta_{\mathscr{H}}^{\alpha / 2}(z)} d z \leq c R .
$$

Now, let us consider III. We have

$$
I I I \leq P^{x}\left(X\left(\tau_{\Phi(-\infty,-2 l)}\right) \in \Phi[-2 l,-l)\right) \sup _{y \in \Phi[-2 l,-l)} P^{y}\left(X\left(\tau_{\mathscr{H}}\right) \in V_{2}\right) .
$$

The first factor can be bounded using Lemma 4.2:

$$
P^{x}\left(X\left(\tau_{\Phi(-\infty,-2 l)}\right) \in \Phi[-2 l,-l)\right) \leq c(\alpha, d) l^{-d-\alpha+1} .
$$

For any $y \in \Phi[-2 l,-l)$ we get, from (2.5),

$$
P^{y}\left(X\left(\tau_{\mathscr{H}}\right) \in V_{2}\right) \leq c(\alpha, d) \int_{V_{2}} \frac{l^{\alpha / 2} d z}{|y-z|^{d} \delta_{\mathscr{H}}^{\alpha / 2}(z)} \leq \frac{c}{l^{d-\alpha / 2}} \int_{V_{2}} \frac{d z}{\delta_{\mathscr{H}}^{\alpha / 2}(z)} .
$$

Recall that $d \geq 2, \alpha<2$ and $l \geq 1 / 3$. This implies that $l^{-2 d-\alpha / 2+1} \leq c(\alpha, d) l^{-d-\alpha}$. It follows that

$$
\begin{aligned}
I I I & \leq c(\alpha, d) \frac{l^{-d-\alpha+1}}{l^{d-\alpha / 2}} \int_{V_{2}} \frac{d z}{\delta_{\mathscr{H}}^{\alpha / 2}(z)} \\
& \leq \frac{c}{l^{d+\alpha}} \int_{V_{2}} \frac{d z}{\delta_{\mathcal{H}}^{\alpha / 2}(z)} \leq c \int_{V_{2}} \frac{d z}{|x-z|^{d+\alpha} \delta_{\mathscr{H}}^{\alpha / 2}(z)} \leq c R .
\end{aligned}
$$

The proof is complete. 
We will assume from now on that $I(f)<\infty$. Let $g(x)=2 f(x)$ and note that $I(g)<\infty$. If for all $x \in(0,1 / 2]$ we have $g(x)<x / 2$ then we set $K_{1}=2$. Otherwise we set

$$
K_{1}=\sup \left\{k \in \mathbf{N}: k \geq 2, \exists x \in\left(2^{-k}, 2^{-k+1}\right] \text { such that } g(x) \geq x / 2\right\} .
$$

It is elementary to check that the condition $I(g)<\infty$ implies that $K_{1}<\infty$. In the sequel we will use the fact that $g(x)<x / 2$ for all $x \in\left(0,2^{-K_{1}}\right]$.

For $l \in \mathbf{N}_{+}$we put

$$
\begin{aligned}
W(l) & =\left\{x \in D(g): x_{1} \in\left(0,2^{-l}\right)\right\}, \\
V(l) & =\left\{x \in D(g): x_{1} \in\left[2^{-l-1}, 2^{-l}\right)\right\}, \\
U(l) & =\left\{x \in \mathbf{R}^{d}: x_{1} \in\left(0,2^{-l}\right),|\tilde{x}|<g\left(2^{-l}\right)\right\}, \\
\widehat{U}(l) & =\left\{x \in \mathbf{R}^{d}: x_{1} \in\left(0,2^{-l-1}\right),|\tilde{x}|<g\left(2^{-l}\right)\right\} .
\end{aligned}
$$

For $l, n \in \mathbf{N}_{+}, n \geq l$, let

$$
W(l, n)=W(l) \cup U(n) .
$$

Note that the sets $W(l), V(l)$ and $U(l)$ are similar to but not identical to sets denoted by the same letters in Section 3.

Recall our convention concerning the constants-they can change their values from line to line. We will need two constants whose values cannot change in this way. To emphasize the different nature of these genuine constants, we will denote them $\tilde{c}_{1}$ and $\tilde{c}_{2}$. They will appear for the first time in Lemmas 4.4 and 4.5.

LEMMA 4.4. For any $k, l \in \mathbf{N}_{+}, k \geq K_{1}$, and $x \in \widehat{U}(l)$ we have

$$
P^{x}\left(X\left(\tau_{U(l)}\right) \in V(k)\right) \leq \tilde{c}_{1}(\alpha, d) g^{\alpha}\left(2^{-l}\right) g^{d-1}\left(2^{-k}\right)\left(2^{-k}\right)^{-\alpha-d+1} .
$$

It follows that for $k, l$ as above, $n \in \mathbf{N}_{+}, n>l$ and $x \in W(l+1, n)$ we have,

$$
P^{x}\left(X\left(\tau_{W(l, n)}\right) \in V(k)\right) \leq \tilde{c}_{1}(\alpha, d) g^{\alpha}\left(2^{-l}\right) g^{d-1}\left(2^{-k}\right)\left(2^{-k}\right)^{-\alpha-d+1} .
$$

The last inequality is also true for $k, l$ as above, $n=l$ and $x \in \widehat{U}(n)$.

PROOF. We begin by proving the first inequality. Note that for $l \leq k$ we have $V(k) \subset U(l)$ so $P^{x}\left(X\left(\tau_{U(l)}\right) \in V(k)\right)=0$. Therefore we can and do assume that $l>k$.

Let $v=\left(2^{-l}, 0, \ldots, 0\right)$. By Lemma 4.3,

$$
\begin{aligned}
P^{x}\left(X\left(\tau_{U(l)}\right) \in V(k)\right) & \leq P^{x-v}\left(X\left(\tau_{\mathcal{C}\left(g\left(2^{-l}\right)\right)}\right) \in V(k)-v\right) \\
& \leq c(\alpha, d) \int_{V(k)-v} \frac{g^{\alpha}\left(2^{-l}\right)}{|x-v-z|^{d+\alpha}}\left(\frac{g^{\alpha / 2}\left(2^{-l}\right)}{\delta_{\mathscr{H}}^{\alpha / 2}(z)} \vee 1\right) d z \\
& \leq c g^{\alpha}\left(2^{-l}\right)\left(2^{-k}\right)^{-\alpha-d} \int_{V(k)-v}\left(\frac{g^{\alpha / 2}\left(2^{-l}\right)}{\delta_{\mathscr{H}}^{\alpha / 2}(z)} \vee 1\right) d z .
\end{aligned}
$$


For $k=l-1$, direct calculations show that the last integral is bounded from above by $c(\alpha, d) 2^{-l+1} g^{d-1}\left(2^{-l+1}\right)$. For $k<l-1$, the last integral is bounded from above by $m(V(k))$. These estimates easily imply the first inequality in the statement of the lemma.

Since $W(l, n) \subset U(l), W(l+1, n) \subset \widehat{U}(l)$ for $n>l$, and $W(n, n)=U(n)$, the second inequality in the lemma is a direct consequence of the first one.

For any $n \in \mathbf{N}_{+}$we will define inductively a sequence of stopping times $S(m, n)$, starting with $S(0, n)=0$ and $S(1, n)=\tau_{W(n, n)}$. For any $m, n \in \mathbf{N}_{+}$we define

$$
S(m+1, n)= \begin{cases}\tau_{W(i-1, n),} & \text { if } X(S(m, n)) \in V(i), \text { for some } i \geq 2, i \in \mathbf{N}, \\ S(m, n), & \text { if } X(S(m, n)) \notin W(2) .\end{cases}
$$

The following lemma gives an estimate for the probability that the process starting from $x \in \widehat{U}(n)$ will end up in $V(k)$ at time $S(m, n)$, and, moreover, $0<S(1, n)<\cdots<S(m, n)$.

LEMMA 4.5. Let $K_{0}=K_{0}(\alpha, d, g) \in \mathbf{N}$ be the smallest constant satisfying $K_{0} \geq K_{1}$ and

$$
J\left(g, K_{0}\right):=\tilde{c}_{1} \sum_{j=K_{0}}^{\infty} \frac{\left(g\left(2^{-j+1}\right)\right)^{d+\alpha-1}}{\left(2^{-j}\right)^{d+\alpha-1}}<\frac{1}{2},
$$

where $\tilde{c}_{1}=\tilde{c}_{1}(\alpha, d)$ is the constant from Lemma 4.4. For any $m \in \mathbf{N}_{+}, n \geq k \geq K_{0}$, $n, k \in \mathbf{N}$ and $x \in \widehat{U}(n)$ we have

$$
P^{x}(S(m-1, n)<S(m, n) ; X(S(m, n)) \in V(k)) \leq \tilde{c}_{2} g^{\alpha}\left(2^{-n}\right) 2^{-m},
$$
where $\tilde{c}_{2}=2 \tilde{c}_{1} g^{d-1}\left(2^{-k}\right)\left(2^{-k}\right)^{-\alpha-d+1}$.

Proof. The constant $K_{0}$ is finite because $I(g)<\infty$ (see Lemma 2.1).

First note that for $m \in \mathbf{N}_{+}$, we have $P^{x}(X(S(m, n)) \in V(n))=0$. This follows from the fact that $S(m, n)=\tau_{W(j, n)}$ for some $j \leq n, j \in \mathbf{N}_{+}$and $V(n) \subset W(j, n)$ ( $j$ may depend on $x, \omega$ and $m$ ). This gives (4.4) for $k=n$. So from now on we will assume that $k \leq n-1$.

We will prove (4.4) by induction on $m$. For $m=1$, (4.4) follows from the second inequality in Lemma 4.4 for $l=n$.

Suppose we have proved (4.4) for some $m \in \mathbf{N}_{+}$and all $n, k \in \mathbf{N}, n-1 \geq$ $k \geq K_{0}$. We will show (4.4) for $m+1$. For any $n, k \in \mathbf{N}, n-1 \geq k \geq K_{0}$, and $x \in \widehat{U}(n)$ we have

$$
\begin{array}{r}
P^{x}(S(m, n)<S(m+1, n) ; X(S(m+1, n)) \in V(k)) \\
=\sum_{j=k}^{n-1} E^{x}(S(m-1, n)<S(m, n) ; X(S(m, n)) \in V(j) ; \\
\left.P^{X(S(m, n))}\left(X\left(\tau_{W(j-1, n)}\right) \in V(k)\right)\right) .
\end{array}
$$


By our induction hypothesis and Lemma 4.4 this is bounded from above by

$$
\begin{aligned}
& \sum_{j=k}^{n-1} 2 \tilde{c}_{1} \frac{g^{d-1}\left(2^{-j}\right)}{\left(2^{-j}\right)^{\alpha+d-1}} g^{\alpha}\left(2^{-n}\right) 2^{-m} \frac{\tilde{c}_{1} g^{d-1}\left(2^{-k}\right) g^{\alpha}\left(2^{-j+1}\right)}{\left(2^{-k}\right)^{\alpha+d-1}} \\
& \quad=2 \tilde{c}_{1} g^{\alpha}\left(2^{-n}\right) 2^{-m} \frac{g^{d-1}\left(2^{-k}\right)}{\left(2^{-k}\right)^{\alpha+d-1}} \sum_{j=k}^{n-1} \frac{\tilde{c}_{1} g^{d-1}\left(2^{-j}\right) g^{\alpha}\left(2^{-j+1}\right)}{\left(2^{-j}\right)^{\alpha+d-1}} \\
& \leq \tilde{c}_{2} g^{\alpha}\left(2^{-n}\right) 2^{-m} \sum_{j=K_{0}}^{\infty} \frac{\tilde{c}_{1} g^{\alpha+d-1}\left(2^{-j+1}\right)}{\left(2^{-j}\right)^{\alpha+d-1}} \\
& \leq \tilde{c}_{2} g^{\alpha}\left(2^{-n}\right) 2^{-m-1} .
\end{aligned}
$$

The last inequality is due to (4.3). We have shown that (4.4) holds for $m+1$.

Now we will define inductively stopping times $S(m, n, x)$. They are "translated" versions of $S(m, n)$ 's so their definition is analogous to the definition of $S(m, n)$.

For $n \in \mathbf{N}_{+}$we will write $y_{n}=\left(2^{-n-2}, 0, \ldots, 0\right) \in \mathbf{R}^{d}$. For $x \in \mathbf{R}^{d}, n \in \mathbf{N}_{+}$we set $S(0, n, x)=0$ and

$$
S(1, n, x)=\tau_{x-y_{n}+W(n, n)}=\inf \left\{t \geq 0: X(t) \notin x-y_{n}+W(n, n)\right\} .
$$

For $m, n \in \mathbf{N}_{+}$and $x \in \mathbf{R}^{d}$ we define $S(m+1, n, x)$ to be

$$
\tau_{x-y_{n}+W(i-1, n)}=\inf \left\{t \geq 0: X(t) \notin x-y_{n}+W(i-1, n)\right\}
$$

if $X(S(m, n, x)) \in x-y_{n}+V(i)$ for some $i \geq 2, i \in \mathbf{N}$. If $X(S(m, n, x)) \notin$ $x-y_{n}+W(2)$ then we define $S(m+1, n, x)$ to be $S(m, n, x)$.

For $n \geq k, k, n, m \in \mathbf{N}_{+}$, consider the following events:

$$
H(n, m, k, x)=\left\{S(m-1, n, x)<S(m, n, x) ; X(S(m, n, x)) \in x-y_{n}+V(k)\right\} .
$$

LEMMA 4.6. For $n \geq k \geq K_{0}, k, n, m \in \mathbf{N}_{+}, x \in \mathbf{R}^{d}$, we have

$$
P^{x}(H(n, m, k, x)) \leq c(\alpha, d, g, k) g^{\alpha}\left(2^{-n}\right) 2^{-m} \text {. }
$$

Proof. We have $P^{x}(H(n, m, k, x))=P^{y_{n}}\left(H\left(n, m, k, y_{n}\right)\right)$ and $S\left(m, n, y_{n}\right)=$ $S(m, n)$. Also note that $y_{n} \in \widehat{U}(n)$. Therefore Lemma 4.6 is a direct consequence of Lemma 4.5.

LEMMA 4.7. Let $\left\{Z_{i}\right\}_{i=1}^{\infty}$ be i.i.d. random variables such that $Z_{i} \geq 0$, $E\left(Z_{i}\right)>0$ and $E\left(Z_{i}\right)^{4}<\infty$. There exists a constant $c$ depending only on the distribution of $Z_{1}$ such that, for any $M \geq 1, M \in \mathbf{R}$, we have

$$
\sum_{j=1}^{\infty} P\left(\sum_{i=1}^{j} Z_{i} \leq M\right) \leq c M
$$


PROOF. Let $\mu=E Z_{i}$. Let $j_{0}$ be the smallest integer with $j_{0} \mu>2 M$. We have

$$
E\left(\sum_{i=1}^{j}\left(Z_{i}-\mu\right)\right)^{4} \leq j E\left(Z_{i}-\mu\right)^{4}+3 j^{2} E\left(Z_{i}-\mu\right)^{2} \leq c j^{2}
$$

because all terms which contain $E\left(Z_{i}-\mu\right)$ are equal to zero.

For $j \geq j_{0}$,

$$
\begin{aligned}
P\left(\sum_{i=1}^{j} Z_{i} \leq M\right) & \leq P\left(\left|\sum_{i=1}^{j}\left(Z_{i}-\mu\right)\right| \geq j \mu-M\right) \\
& =P\left(\left(\sum_{i=1}^{j}\left(Z_{i}-\mu\right)\right)^{4} \geq(j \mu-M)^{4}\right) \\
& \leq E\left(\sum_{i=1}^{j}\left(Z_{i}-\mu\right)\right)^{4}(j \mu-M)^{-4} \leq c j^{2}(j \mu / 2)^{-4} \leq c j^{-2} .
\end{aligned}
$$

It follows that

$$
\begin{aligned}
\sum_{j=1}^{\infty} P\left(\sum_{i=1}^{j} Z_{i} \leq M\right) & \leq \sum_{j=1}^{j_{0}-1} P\left(\sum_{i=1}^{j} Z_{i} \leq M\right)+\sum_{j=j_{0}}^{\infty} P\left(\sum_{i=1}^{j} Z_{i} \leq M\right) \\
& \leq j_{0}+\sum_{j=j_{0}}^{\infty} c j^{-2} \leq 2 M / \mu+1+2 c
\end{aligned}
$$

This clearly implies the lemma.

For any $n \in \mathbf{N}_{+}$and $x \in \mathbf{R}^{d}$ let

$$
\Lambda(n, x)=x+\left\{z \in \mathbf{R}^{d}: z_{1} \in\left(-2^{-n-2}, 2^{-n-2}\right),|\tilde{z}|<g\left(2^{-n}\right) / 2\right\} .
$$

For $j \in \mathbf{N}$ and $n \in \mathbf{N}_{+}$we define the following stopping times: $T(0, n)=0$,

$$
T(j+1, n)=T(j, n)+\tau_{\Lambda(n, X(0))} \circ \theta_{T(j, n)} .
$$

An alternative but equivalent definition is

$$
T(j+1, n)=\inf \{s>T(j, n): X(s) \notin \Lambda(n, X(T(j, n)))\} .
$$

LEMMA 4.8. For any $N \in \mathbf{N}_{+}, n \geq k \geq K_{0}, k, n, m \in \mathbf{N}_{+}$we have

$$
P\left(\bigcup_{j=0}^{\infty}\left[\{T(j, n) \leq N\} \cap \theta_{T(j, n)}^{-1} H(n, m, k, X(0))\right]\right) \leq c(\alpha, d, g, k) N 2^{-m} .
$$

We also have $P\left(\lim _{j \rightarrow \infty} T(j, n)<\infty\right)=0$ for $n \in \mathbf{N}_{+}$. 
PROOF. By the strong Markov property applied at $T(j, n)$ and Lemma 4.6,

$$
\begin{aligned}
& P\left(\{T(j, n) \leq N\} \cap \theta_{T(j, n)}^{-1} H(n, m, k, X(0))\right) \\
& \quad \leq c(\alpha, d, g, k) P(T(j, n) \leq N) g^{\alpha}\left(2^{-n}\right) 2^{-m} .
\end{aligned}
$$

Therefore, to prove the first part of the lemma it will be sufficient to show that

$$
\sum_{j=0}^{\infty} P(T(j, n) \leq N) \leq c(\alpha, d) N g^{-\alpha}\left(2^{-n}\right) .
$$

We have $\widehat{T}(j+1, n) \leq T(j+1, n)$ for stopping times $\widehat{T}(j+1, n)$ defined by

$$
\widehat{T}(j+1, n)=T(j, n)+\tau_{B\left(X(0), g\left(2^{-n}\right) / 2\right)} \circ \theta_{T(j, n)}, \quad j \in \mathbf{N} .
$$

Let $Y_{i}=g^{-\alpha}\left(2^{-n}\right) 2^{\alpha}[\widehat{T}(i, n)-T(i-1, n)], i \in \mathbf{N}_{+}$, and note that $\left\{Y_{i}\right\}_{i=1}^{\infty}$ are i.i.d. random variables with the same distribution as $\tau_{B(X(0), 1)}$. We have

$$
\begin{aligned}
\sum_{j=0}^{\infty} P(T(j, n) \leq N) & \leq 1+\sum_{j=1}^{\infty} P\left(\sum_{i=1}^{j}(T(i, n)-T(i-1, n)) \leq N\right) \\
& \leq 1+\sum_{j=1}^{\infty} P\left(\sum_{i=1}^{j}(\widehat{T}(i, n)-T(i-1, n)) \leq N\right) \\
& =1+\sum_{j=1}^{\infty} P\left(\sum_{i=1}^{j} Y_{i} \leq g^{-\alpha}\left(2^{-n}\right) 2^{\alpha} N\right) .
\end{aligned}
$$

It is easy to show that $E\left(Y_{i}\right)^{4}<\infty$ using (2.1). So we can apply Lemma 4.7 to estimate the right-hand side of (4.6). This gives (4.5).

The last claim in the lemma follows easily from the fact that the series in (4.5) is convergent for every fixed $N$.

Let

$$
\begin{array}{r}
\Omega_{1}=\left\{\omega \in \Omega: \exists s=s(\omega) \geq 0, \exists t_{1}=t_{1}(\omega)>0\right. \text { such that } \\
\left.X(t, \omega)=X(s, \omega) \text { for all } t \in\left[s, s+t_{1}\right)\right\} .
\end{array}
$$

We have

$$
P\left(\Omega_{1}\right)=0,
$$

because for every pair of rationals $s_{1} \neq s_{2}, P\left(X\left(s_{1}\right) \neq X\left(s_{2}\right)\right)=1$.

Proof of Theorem 1.1(ii). For any $n \in \mathbf{N}_{+}$and $x \in \mathbf{R}^{d}$ let

$$
U(n, x)=x+\left\{z \in \mathbf{R}^{d}: z_{1} \in\left(-2^{-n}, 2^{-n}\right),|\tilde{z}|<g\left(2^{-n}\right)\right\} .
$$


For $s \geq 0$ and $n \in \mathbf{N}_{+}$we define stopping times

$$
Q(s, n)=\inf \{t>s: X(t) \notin U(n, X(s))\} .
$$

By the right continuity of paths we obtain $Q(s, n)>s$ for all $s$. For $\omega \in \Omega \backslash \Omega_{1}$ we also have $\lim _{n \rightarrow \infty} Q(s, n, \omega)=s$ and $\lim _{n \rightarrow \infty} X(Q(s, n, \omega))=X(s, \omega)$ for all $s$.

Next, let us write $\widehat{U}(n, x)=U(n, x) \backslash U(n+1, x), n \in \mathbf{N}_{+}, x \in \mathbf{R}^{d}$, and for $\omega \in \Omega \backslash \Omega_{1}, s \geq 0$ and $t>0$ define

$$
\begin{array}{r}
Z(s, t, \omega)=\left\{l \in \mathbf{N}_{+}: \exists a \in \mathbf{N}_{+} \text {such that } Q(s, a, \omega) \in(s, s+t),\right. \\
X(Q(s, a, \omega), \omega) \in \widehat{U}(l, X(s, \omega))\} .
\end{array}
$$

Our remarks about $Q(s, n)$ imply that for all $\omega \in \Omega \backslash \Omega_{1}, s \geq 0$ and $t>0$ the number of elements of $Z(s, t, \omega)$ is infinite.

Let $\Omega_{2}=\Omega_{1} \cup \bigcup_{n=1}^{\infty}\left\{\lim _{j \rightarrow \infty} T(j, n)<\infty\right\}$. By Lemma 4.8 and (4.7) we have $P\left(\Omega_{2}\right)=0$.

Recall that $X^{(1)}(t), \ldots, X^{(d)}(t)$ denote the components of $X(t)$. We will write $D(f, k)=\left\{x \in D(f): x_{1} \in\left(0,2^{-k}\right)\right\}, k \in N$. For any $N \in \mathbf{N}_{+}$and $k \geq K_{0}+1$, $k \in \mathbf{N}$, let us denote

$$
\begin{aligned}
A(f,[0, N], k)=\left\{\omega \in \Omega \backslash \Omega_{2}: \exists s=s(\omega) \in[0, N], \exists t_{1}=t_{1}(\omega)>0\right. \text { such that } \\
\\
\text { (i) } X(t, \omega) \in X(s, \omega)+\bar{D}\left(f, K_{0}+2\right) \text { for all } t \in\left[s, s+t_{1}\right), \\
\text { (ii) } \left.\sup _{t \in\left[s, s+t_{1}\right)}\left|X^{(1)}(t, \omega)-X^{(1)}(s, \omega)\right| \in\left[2^{-k-1}, 2^{-k}\right)\right\} .
\end{aligned}
$$

Recall $A(f)$ and a fixed time $t_{0}>0$ from the Introduction. By the right continuity of paths we have

$$
A(f) \subset \Omega_{2} \cup \bigcup_{N=1}^{\infty} \bigcup_{k=K_{0}+1}^{\infty} A(f,[0, N], k) .
$$

To prove Theorem 1.1(ii) it will suffice to show that for any $N \in \mathbf{N}_{+}$and $k \geq$ $K_{0}+1, k \in \mathbf{N}$, we have $P(A(f,[0, N], k))=0$.

Fix $N$ and $k$. For $s \geq 0, t>0, \omega \in \Omega \backslash \Omega_{1}$ and $i>k, i \in \mathbf{N}$, we will write

$$
Z(s, t, i, k, \omega)=Z(s, t, \omega) \cap\{k, k+1, \ldots, i-1\} .
$$

Let \# denote the number of elements of a set. For $i>k, m \in \mathbf{N}_{+}$and $\Gamma=[0, N]$ or $\Gamma=[0, N] \cap[T(j, n), T(j+1, n)), j \in \mathbf{N}, n \geq i$, let $A(f, \Gamma, i, m, k)=\left\{\omega \in \Omega \backslash \Omega_{2}: \exists s=s(\omega) \in \Gamma, \exists t_{1}=t_{1}(\omega)>0\right.$ such that

(i) $X(t, \omega) \in X(s, \omega)+\bar{D}\left(f, K_{0}+2\right)$ for all $t \in\left[s, s+t_{1}\right)$,

(ii) $\sup _{t \in\left[s, s+t_{1}\right)}\left|X^{(1)}(t, \omega)-X^{(1)}(s, \omega)\right| \in\left[2^{-k-1}, 2^{-k}\right)$,

(iii) $\left.\# Z\left(s, t_{1}, i, k, \omega\right) \geq m\right\}$. 
We claim that, if

$$
\omega \in A(f,[0, N] \cap[T(j, n), T(j+1, n)), i, 3 m, k),
$$

and $j, n, i \in \mathbf{N}, n \geq i>k, m \in \mathbf{N}_{+}$, then

$$
\omega \in\{T(j, n) \leq N\} \cap \theta_{T(j, n)}^{-1}\left[\bigcup_{l=m}^{\infty} \bigcup_{b=k-1}^{k+1} H(n, l, b, X(0))\right] .
$$

To show that the above claim is true is straightforward but tedious so we defer the argument to the end of the proof.

Assume that (4.8) implies (4.9). If $\omega \in A(f,[0, N], i, 3 m, k)$ then

$$
\omega \in F(n, m, k):=\bigcup_{j=0}^{\infty}\left[\{T(j, n) \leq N\} \cap \theta_{T(j, n)}^{-1}\left[\bigcup_{l=m}^{\infty} \bigcup_{b=k-1}^{k+1} H(n, l, b, X(0))\right]\right] .
$$

Consequently,

$$
\bigcup_{i=k+1}^{\infty} A(f,[0, N], i, 3 m, k) \subset \liminf _{n \rightarrow \infty} F(n, m, k) .
$$

Lemma 4.8 easily implies that

$$
P(F(n, m, k)) \leq c(\alpha, d, f, k) N 2^{-m}, \quad n>k, n, m \in \mathbf{N}_{+} .
$$

Since for any $s \geq 0, t>0$ and $\omega \in \Omega \backslash \Omega_{1}$ the set $Z(s, t, \omega)$ has infinitely many elements, we have

$$
A(f,[0, N], k) \subset \bigcap_{m=1}^{\infty} \bigcup_{i=k+1}^{\infty} A(f,[0, N], i, 3 m, k) .
$$

From (4.11) we obtain $P\left(\bigcap_{m=1}^{\infty} \liminf _{n \rightarrow \infty} F(n, m, k)\right)=0$. This, (4.10), (4.11) and (4.12) imply that $P(A(f,[0, N], k))=0$. Consequently, $P(A(f))=0$.

Now what is left is to prove that (4.8) implies (4.9). Assume that $\omega$ satisfies (4.8). Then there exist $s=s(\omega) \in[T(j, n), T(j+1, n))$ and $t_{1}=t_{1}(\omega)>0$ such that $X(t, \omega) \in X(s, \omega)+\left\{x \in \bar{D}(f): x_{1} \in\left[0,2^{-k}\right)\right\}$ for all $t \in\left[s, s+t_{1}\right)$. This follows from conditions (i) and (ii) in the definition of $A(f, \Gamma, i, 3 m, k)$. We will define $G_{1}$-jumps and, later, $G_{2}$-jumps. Here $G$ stands for "good" and subscripts indicate two different but closely related types of jumps. A $G_{1}$-jump occurs if at the first time $t \in\left[s, s+t_{1}\right)$ when the path $X(\cdot, \omega)$ leaves

$$
\begin{gathered}
U\left(q_{1}, X(s, \omega)\right) \cap\left(X(s, \omega)+\left\{x \in \bar{D}(f): x_{1} \in\left[0,2^{-k}\right)\right\}\right) \\
=X(s, \omega)+\left\{x \in \bar{D}(f): x_{1} \in\left[0,2^{-q_{1}}\right)\right\}
\end{gathered}
$$

it jumps to

$$
\begin{aligned}
\widehat{U}\left(q_{2}, X(s, \omega)\right) & \cap\left(X(s, \omega)+\left\{x \in \bar{D}(f): x_{1} \in\left[0,2^{-k}\right)\right\}\right) \\
& =X(s, \omega)+\left\{x \in \bar{D}(f): x_{1} \in\left[2^{-q_{2}-1}, 2^{-q_{2}}\right)\right\},
\end{aligned}
$$


for some particular $q_{1}, q_{2} \in \mathbf{N}_{+}$, which are chosen below. We say that the first $G_{1}$-jump occurs if $q_{1} \geq i$ and $i>q_{2} \geq k$. For the "consecutive" $G_{1}$-jumps, the corresponding condition is $i-1 \geq q_{1}>q_{2}>k$. Note that the numbers $q_{1}$ and $q_{2}$ are chosen so that $G_{1}$-jumps corrrespond to $Z\left(s, t_{1}, i, k, \omega\right)$.

The fact that $\omega$ satisfies (4.8) implies that for $t \in\left[s, s+t_{1}\right)$, the path $X(t, \omega)$ has at least $3 m G_{1}$-jumps.

Next we define a $G_{2}$-jump. We will say that the first $G_{2}$-jump occurs if at the first time $t>T(j, n)$ when the path $X(\cdot, \omega)$ leaves the set $X(T(j, n))-y_{n}+$ $W(n, n)$, it jumps to

$$
X(T(j, n))-y_{n}+V(q)=X(T(j, n))-y_{n}+\left\{x \in D(g): x_{1} \in\left[2^{-q-1}, 2^{-q}\right)\right\}
$$

for some $q \in \mathbf{N}_{+}, n>q \geq b$. We will say that consecutive $G_{2}$-jumps occur if for some $q_{1}, q_{2} \in \mathbf{N}_{+}, n>q_{1}>q_{2} \geq b, q_{1}-q_{2}>1$, at the first time $t>T(j, n)$ when the path $X(\cdot, \omega)$ leaves

$$
\begin{aligned}
& X(T(j, n))-y_{n}+W\left(q_{1}-1, n\right) \\
& \quad=X(T(j, n))-y_{n}+\left(\left\{x \in D(g): x_{1} \in\left(0,2^{-q_{1}+1}\right)\right\} \cup U(n)\right),
\end{aligned}
$$

it jumps to

$$
X(T(j, n))-y_{n}+V\left(q_{2}\right)=X(T(j, n))-y_{n}+\left\{x \in D(g): x_{1} \in\left[2^{-q_{2}-1}, 2^{-q_{2}}\right)\right\} .
$$

$G_{2}$-jumps correspond to $H(n, l, b, X(0))$ and $S(l, n, X(0))$.

If $\omega$ satisfies (4.9) then for $t>T(j, n)$, the path $X(t, \omega)$ has at least $m$ $G_{2}$-jumps.

Recall we have assumed that $\omega$ satisfies (4.8) and let

$$
z=\left(z_{1}, z_{2}, \ldots, z_{d}\right)=X(s, \omega)-\left(X(T(j, n))-y_{n}\right) .
$$

Since $s \in[T(j, n), T(j+1, n))$ the vector $X(s, \omega)-X(T(j, n))$ belongs to $\Lambda(n, 0)$, so in particular $|\tilde{z}|=\left|\left(z_{2}, \ldots, z_{d}\right)\right|<g\left(2^{-n}\right) / 2=f\left(2^{-n}\right)$. Put $z^{\prime}=(0, \tilde{z})$ and $z^{\prime \prime}=\left(z_{1}, 0, \ldots, 0\right)$. We may think of $z^{\prime}$ as the vertical part of the vector $z$ and of $z^{\prime \prime}$ as the horizontal part. Recall that $W(n, n)=U(n)=\left\{x \in \mathbf{R}^{d}: x_{1} \in\right.$ $\left.\left(0,2^{-n}\right),|\tilde{x}|<g\left(2^{-n}\right)\right\}$.

For $s \in[T(j, n), T(j+1, n))$, we have

$$
\begin{aligned}
& X(s, \omega) \in \Lambda(n, X(T(j, n))) \\
& X(s, \omega)+\bar{D}\left(f, K_{0}+2\right) \subset X(T(j, n))-y_{n}+U(n), \\
& X(j, n)-y_{n}+\left(D\left(g, K_{0}\right) \cup U(n)\right) .
\end{aligned}
$$

We also have

$$
\begin{aligned}
& z^{\prime}+X(T(j, n))-y_{n}+\left\{x \in \bar{D}(f): x_{1} \in\left(0,2^{-q}\right)\right\} \\
& \quad \subset X(T(j, n))-y_{n}+\left(\left\{x \in D(g): x_{1} \in\left(0,2^{-q}\right)\right\} \cup U(n)\right),
\end{aligned}
$$


for $q \in \mathbf{N}_{+}, n \geq q$ and

$$
\begin{aligned}
z^{\prime}+ & X(T(j, n))-y_{n}+\left\{x \in \bar{D}(f): x_{1} \in\left[2^{-q-1}, 2^{-q}\right)\right\} \\
& \subset X(T(j, n))-y_{n}+\left\{x \in D(g): x_{1} \in\left[2^{-q-1}, 2^{-q}\right)\right\},
\end{aligned}
$$

for $q \in \mathbf{N}_{+}, n-1 \geq q$. It follows from (4.13)-(4.16) that (4.8) implies (4.9), except for two fine points of the argument, which are discussed below.

Let us denote $\tilde{D}(q)=\left\{x \in \bar{D}(f): x_{1} \in\left[2^{-q-1}, 2^{-q}\right)\right\}$ and recall $V(q)=\{x \in$ $\left.D(g): x_{1} \in\left[2^{-q-1}, 2^{-q}\right)\right\}, q \in \mathbf{N}$.

(i) There is a difference between $G_{1}$-jumps and $G_{2}$-jumps. Suppose that during some $G_{1}$-jump, the process jumps to the set $X(s, \omega)+\tilde{D}(q)$. Then during the next $G_{1}$-jump, the process may jump to the "nearest neighbor" of this set, that is, the set $X(s, \omega)+\tilde{D}(q-1)$. On the other hand if during some $G_{2}$-jump the process jumps to the set $X(T(j, n))-y_{n}+V(q)$ then during the next $G_{2}$-jump the process cannot jump to the "nearest neighbor" of this set, that is, the set $X(T(j, n))-y_{n}+V(q-1)$. We indicate how to resolve this problem below.

(ii) Recall that $z^{\prime \prime}=\left(z_{1}, 0, \ldots, 0\right)$ is the horizontal part of the vector $z=$ $X(s, \omega)-\left(X(T(j, n))-y_{n}\right)$. There is a "small horizontal translation" by the vector $z^{\prime \prime}$ between the sets $X(s, \omega)+\tilde{D}(q)$ and $X(T(j, n))-y_{n}+V(q)$. Since the vector $X(s, \omega)-X(T(j, n))$ belongs to $\Lambda(n, 0)$ we have $\left|z^{\prime \prime}\right|<2^{-n-2}+\left|y_{n}\right|=$ $2^{-n-1}$ so the vector $z^{\prime \prime}$ is relatively small in comparison to the sets $\tilde{D}(q)$ and $V(q)$ for $n-1 \leq q$.

The way we can deal with problems in (i) and (ii) is by having at least $3 \mathrm{~m}$ $G_{1}$-jumps in (4.8). This guaranties, in spite of the difference between $G_{1}$-jumps and $G_{2}$-jumps and the "small horizontal translations," that the path $X(t, \omega)$ has at least $m G_{2}$-jumps.

Similarly in (4.9) we consider an auxiliary parameter $b=k-1, k$ or $k+1$. This parameter is chosen so that the condition (ii) in the definition of $A(f, \Gamma, i, 3 m, k)$ implies that after one of the $G_{2}$-jumps, the path is in $X(T(j, n))-y_{n}+V(b)$ (for $b=k-1, k$ or $k+1)$.

Acknowledgments. We are grateful to David White and the referee for careful reading of the manuscript and many helpful suggestions.

\section{REFERENCES}

[1] Adelman, O., Burdzy, K. and Pemantle, R. (1998). Sets avoided by Brownian motion. Ann. Probab. 26 429-464.

[2] Bass, R. and Burdzy, K. (1999). Cutting Brownian Paths. Amer. Math. Soc., Providence, RI.

[3] Bertoin, J. (1996). Lévy Processes. Cambridge Univ. Press.

[4] Blumenthal, R. M. and Getoor, R. K. (1968). Markov Processes and Potential Theory. Academic Press, New York.

[5] Blumenthal, R. M., Getoor, R. K. and Ray, D. B. (1961). On the distribution of first hits for the symmetric stable processes. Trans. Amer. Math. Soc. 99 540-554. 
[6] Bogdan, K. (1999). Representation of $\alpha$-harmonic functions in Lipschitz domains. Hiroshima Math. J. 29 227-243.

[7] Bogdan, K., Burdzy, K. and Chen, Z.-Q. Censored stable processes. Preprint.

[8] Bogdan, K. and Byczkowski, T. (1999). Potential theory for the $\alpha$-stable Schrödinger operator on bounded Lipschitz domains. Studia Math. 133 53-92.

[9] BogdAN, K. and BYCZKOWSKI, T. (1999). Probabilistic proof of boundary Harnack principle for $\alpha$-harmonic functions. Potential Anal. 11 135-156.

[10] BurdZy, K. (1985). Brownian paths and cones. Ann. Probab. 13 1006-1010.

[11] Chen, Z.-Q. (1999). Multidimensional symmetric stable processes. Korean J. Comput. Appl. Math. 6 227-266.

[12] Davis, B. (1983). On Brownian slow points. Z. Wahrsch. Verw. Gebiete 64 359-367.

[13] Dvoretzky, A. (1963). On the oscillation of the Brownian motion process. Israel J. Math. 1 212-214.

[14] Evans, S. N. (1985). On the Hausdorff dimension of Brownian cone points. Math. Proc. Cambridge Philos. Soc. 98 343-353.

[15] Greenwood, P. and Perkins, E. (1983). A conditioned limit theorem for random walk and Brownian local time on square root boundaries. Ann. Probab. 11 227-261.

[16] IKEDA, N. and WatAnabe, S. (1962). On some relations between the harmonic measure and the Lévy measure for a certain class of Markov processes. J. Math. Kyoto Univ. 2 79-95.

[17] JAFFARD, S. (1999). The multifractal nature of Lévy processes. Probab. Theory Related Fields $114207-227$.

[18] Le Gall, J.-F. (1987). Mouvement brownien, cônes et processus stables. Probab. Theory Related Fields 76 587-627.

[19] Shimura, M. (1985). Excursions in a cone for two-dimensional Brownian motion. J. Math. Kyoto Univ. 25 433-443.

DEPARTMENT OF MATHEMATICS

UNIVERSITY OF WASHINGTON

BOX 354350

SEATtLE, WASHINGTON 98195-4350

E-MAIL: burdzy@math.washington.edu

\author{
InSTITUTE OF MATHEMATICS \\ Polish ACADEMy OF SCIENCES \\ UL. KOPERNIKA 18 \\ 51-617 WROCŁAW \\ POLAND \\ AND \\ INSTITUTE OF MATHEMATICS \\ WROCŁAW UNIVERSITY OF TECHNOLOGY \\ UL. WYBRZEŻE WYSPIAŃSKIEGO 27 \\ 50-370 WROCŁAW \\ POLAND \\ E-MAIL: tkulczyc@kac.im.pwr.wroc.pl
}

